\title{
LAS FUNCIONES DE LA TOLERANCIA EN LA RESOCIALIZACIÓN POLÍTICA DE LA CIUDADANÍA
}

\author{
Gustavo FONDEVILA ${ }^{1}$
}

- RESUMEN: Este trabajo analiza críticamente la caracterización del comunitarismo (Michael Walzer y Amitai Etzioni) respecto de los problemas de desintegración social de las sociedades post-industriales (diagnóstico social) y las categorías propuestas (tolerancia, solidaridad y resocialización moral) para solucionar dichos problemas y sus consecuencias (apatía ciudadana, desinterés por los asuntos públicos, falta de compromiso social, criminalidad, vandalismo, etc.) El análisis se centra en el carácter funcional que asume el modelo de integración social comunitarista y en los problemas que presentan los fundamentos de ese modelo (solidaridad y tolerancia).

- PALABRAS-CLAVE: Comunitarismo; resocialización; tolerancia; solidaridad; integración social.

El comunitarismo surgió en la década del 80' como contrapartida teórica a la revitalización del liberalismo político promovida por la Theory of Justice de John Rawls (1971). El comunistarismo ofrecía frente al minimalismo de justicia una concepción completa de lo bueno, al proponer la recuperación de las ideas de bien y virtud en el contexto de las comunidades. Según la corriente republicana, la ética de la justicia debe complementarse con una ética de la autenticidad, de lealtad a la identidad comunitaria (ver Taylor, 1989 e 1991).

1 Institut für Sozialwissenschaften/Universität Alexander von Humboldt zu Berlin/Alemania. 
Estas críticas al liberalismo que comenzaron a dominar la teoría política en USA durante la década de 1980, adquirieron relieve filosófico y peso político propio. $\mathrm{Y}$ en muchos casos, sirvieron directamente para inspirar políticas de gobierno o al menos, discursos de campaña, y explicar acontecimientos políticos y sociales de importancia. Por ejemplo, el best-seller de Etzioni, The Spirit of Community cruzó el atlántico y fue adoptado por Tony Blair y los defensores del llamado Third Way. En la actualidad, el llamado a los valores comunitarios, el reempowerment de la civil society y la retirada del Estado social, forman también parte habitual del discurso de la socialdemocracia en USA y también, por ejemplo, del canciller alemán Gerhard Schröder (Tagesspiegel, 1999, p.8).

En el terreno de las ciencias sociales y de la teoría política, el comunitarismo también ganó en importancia y extensión internacional. Hoy en día, por ejemplo, en Alemania es casi imposible pensar en una publicación universitaria, seminario o discusión en el terreno de las ciencias sociales o filosofía que no mencione el debate comunitarista.

En este sentido, este trabajo pretende analizar críticamente la caracterización del comunitarismo respecto de los problemas de desintegración social de las sociedades post-industriales (diagnóstico social) y las categorías propuestas (tolerancia, solidaridad y resocialización moral) para solucionar dichos problemas y sus consecuencias (apatía ciudadana, desinterés por los asuntos públicos, falta de compromiso social, criminalidad, vandalismo etc.). El análisis se centra en el carácter funcional que asume el modelo de integración social comunitarista y en los problemas que presentan los fundamentos de ese modelo (solidaridad y tolerancia). ${ }^{2}$

En 1980, Michael Walzer (p.23-53) publicó un texto en el que analizaba las posibilidades políticas de establecer una democracia participativa en las llamadas sociedades avanzadas, bajo las condiciones de una creciente política de bienestar social instrumentada por el Estado. En ese trabajo, diagnosticaba una tensión problemática en relación a la participación política y la justicia social. ${ }^{3}$ Esta tensión tiene que ver con el aumento de funciones del Estado social. La práctica política de dicho Estado, que tiene consecuencias positivas importantes para el balance re-distributivo de bienestar de una sociedad, es decir, para la justicia social de la misma, trajo en su desarrollo histórico efectos negativos. Por un lado, se produjo una desmitificación pragmática de la comprensión del Estado por parte de la ciudadanía y por otro lado, una gran presión

2 El análisis se ocupa sobre todo de la obra de Michael Walzer y en menor medida de Amitai Etzioni. 3 Este tema tiene cierta tradición dentro de la teoría política y crítica social. Ver Bell, 1976. 
auto-legitimatoria canalizada a través de los derechos sociales y los beneficios derivados. Esta búsqueda de legitimación y la instrumentación del poder político a través de los programas sociales significó en términos prácticos, una reducción de la libertad de los recipients de la ayuda social en particular y de la ciudadanía en general.

La dinámica estatal de reconocimiento de nuevos derechos sociales, ha provocado en los países post-industriales que el Estado creciera e incorporara nuevas funciones. Con cada nuevo reconocimiento de una necesidad social, el sistema incorpora una nueva dependencia para satisfacer y aprovechar y se genera una nueva atadura social. Cada vez que el Estado convierte a un ciudadano en un receptor directo de la ayuda social, deja fuera del proceso re-distributivo a las redes solidarias de la sociedad civil, es decir, a los sindicatos, comunidades religiosas, agrupaciones vecinales, grupos privados, fundaciones, etc. De este modo, debilita los lazos sociales naturales de la comunidad al no integrarla al proceso de redistribución (Walzer, 1980, p.32). Los individuos deben mirar exclusivamente hacia el Estado cuando pretenden obtener una reducción o una socialización mayor del riesgo de la economía de mercado. Según esta perspectiva, se promueve la pasividad ciudadana, dado que no es necesario volverse activo para obtener ayuda en el circuito solidario de la comunidad sino que se puede esperarla pasivamente del Estado. Además, de la mano de los programas sociales, el Estado se presenta a sí mismo como la única fuente de los bienes públicos. Con esta política, delimita la independencia de la comunidad y le resta la posibilidad de constituirse en un lugar de prácticas solidarias y de engagement ciudadano. El paternalismo estatal destruye un lugar donde los ciudadanos pueden determinarse políticamente a sí mismos.

Esta crítica del Estado social no significa que el comunitarismo en general y Walzer en particular crean que dicho Estado deba ser reemplazado directamente por la sociedad civil, sino limitado y reformulado de tal modo que integre a las redes sociales propias de la comunidad. La propuesta política de Walzer pasa por restar las pretensiones morales y eliminar el poder político innecesario del Estado para reducirlo a una transparente administración que pueda ser manejada y comprendida en pequeños grupos. Esta estrategia de descentralización y localización de la política de bienestar permitiría contrarrestar la amenaza de exceso de poder estatal y la reducción de la libertad ciudadana. Y por sobre todas las cosas, esta estrategia abriría espacio para el reforzamiento de los elementos integradores de la acción social en el marco de la comunidad. 
Para el comunitarismo, el reforzamiento de la dinámica integradora de la comunidad implica reforzar los lazos solidarios de la misma. Es decir, propone un aumento de la solidaridad como forma de integración social contra las consecuencias negativas de una política de bienestar orientada exclusivamente a la reproducción del poder político del Estado y a la mera distribución instrumental de los recursos financieros destinados a la ayuda social. Con esta crítica, el comunitarismo pretende desmitificar la idea de que democracia y welfare state son sinónimos. Para esta corriente, la única manera de garantizar la democracia no es a través de la ampliación del reconocimiento de nuevas necesidades de los individuos sino a partir de un aumento de sus capacidades para procurarse ayuda por sí mismos y de mostrarse activos para participar en el desarrollo de la comunidad. En este punto, Walzer se aparta conscientemente de la propuesta habermasiana (ver Habermas, 1985) de realizar en sociedades pluralistas una "neue legitime Gewaltenteilung" a través de un ambicioso concepto de una "höherstufigen Solidarität", que permita trascender todas las identidades particulares de los ciudadanos (Krause \& Malowitz, 1998, p.137).

Frente a esto, Walzer opta por recuperar las formas no mediadas de la solidaridad práctica, tal como aparece y es registrada en la cotidiana, silenciosa y regular cooperación particular de la comunidad. El mantenimiento de la democracia y de las formas democráticas de resolver los conflictos sociales no se realiza sólo mediante la institucionalización de normas morales sino a través del compromiso directo de los ciudadanos. Por este motivo, insiste en observar y recuperar la praxis cotidiana de las formas civiles de la acción social y de la resolución de conflictos.

La forma en la que debemos considerar la ciudadanía y la solidaridad es a través de la disposición de ayuda mutua en el contexto de las comunidades particulares. Esta disposición para la ayuda debe ser vivida y practicada a diario en el seno de una comunidad para poder hablar de una solidaridad concreta. Dicha solidaridad establece una obligación con los demás. Una obligación que es aceptada y compartida por todos los miembros de la comunidad en mayor o menor medida. De hecho, esta solidaridad presupone una forma realista y comprometida de obligación constituida al interior de la comunidad. Así entendida, la solidaridad forma una modalidad de integración libre y voluntaria cuya fuerza integradora es insustituible por ninguna legislación o modalidad jurídica coercitiva de integración social.

Ese interés por los demás que se revela en el compromiso en una actividad conjunta constituye una fuerza emocional que sirve para conformar una identidad común. Esa fuerza universalizadora es el funda- 
mento moral de la civilidad y refiere un tipo de conexión ética. La misma está basada en un proceso de socialización que no supone sujetos aislados unos de otros sino una intersubjetividad constituida en el reconocimiento de la particularidad de todos los singulares. La vida pública que se genera en la sociedad civil y que es la base de la civilidad no puede tener vigencia como el resultado de las delimitaciones recíprocas de los espacios de libertad privada sino como realización de la libertad de todos los singulares que participan de esa comunidad a través de la delimitación de la propia esfera de acción en favor de otro. El reconocimiento recíproco y simétrico de la solidaridad es el que permite el surgimiento de la valoración social de los individuos. Al respecto, Frankenberg (1995, p.195) señala que la solidaridad es "a voluntary act of recognizing others as deserving one's esteem, care and support ...". Además, los individuos de una comunidad valoran a los otros como capaces de habilidades de alto contenido social. Esta experiencia de valoración social está unida a la seguridad sentida de poder hacer cosas que son reconocidas por el resto de la comunidad como valiosas. ${ }^{4}$ Esta forma de interacción en la cual cada persona puede contar con el reconocimiento solidario de las demás es la base de formación de la identidad e integración social.

Según Walzer, la praxis cotidiana de la solidaridad demanda que las comunidades la auto-limiten al interior de sí mismas. Para este autor, la comprensión deliberativa de la solidaridad y la visión de un conjunto de comunidades que participan en la esfera pública desarrollando una solidaridad trascendental superadora de diferencias y de las solidaridades particulares de las comunidades resulta irrealista e innecesaria. A diferencia de Sandel o de MacIntyre que piensan en la ciudadanía como una "toma de obligaciones" cuyo ideal es el ciudadano virtuoso que vive en la eticidad común de una esencia comunitaria políticamente integrada, Walzer prefiere pensar en una pluralidad de distintas orientaciones comunitarias, en las cuales, los individuos pueden formar sus gustos y preferencias, volverse políticamente activos y compartir con otros, sus intereses. El rechazo a la concepción republicana basada en la virtud, o la deliberativa que propone una abstracta Gemeinwohlorientierung, significa recuperar las redes locales y los espacios de acción comunitarios por debajo de la dimensión estatal.

La comprensión walzeriana de la sociedad civil como una "esfera de esferas" significa aceptar que dicha sociedad civil tiene fuerza democra-

4 Desde una perspectiva teórica diferente, Honneth (1992) sostiene que esta forma de auto-respeto o autoestima es lo que permite hablar de solidaridad social. 
tizadora y cumple sus objetivos cuando logra que los ciudadanos no sólo se comprometan activamente en la comunidad sino que además persigan intereses comunitarios que trasciendan los personales e individuales. Esos intereses comunitarios se generan a partir de la esencia política comunitaria y tienen la finalidad de proteger las redes de asociaciones. La dirección del comportamiento político de los ciudadanos dentro de la comunidad debe estar regido por la praxis de la tolerancia. En este sentido, una sociedad civil democrática presenta dos condiciones para la participación política de los individuos y las comunidades:

a) Condiciones de posibilidad: la sociedad civil debe ofrecer un lugar de acción libre para que los individuos puedan perseguir sus propios intereses y realizar sus objetivos particulares. Las actividades pueden estar dirigidas a la protección o promoción de un determinado patrimonio cultural o de una tradición religiosa, etc. Dichas actividades pueden diferir entre sí pero deben reconocerse y tolerarse mutuamente. Esta acción refuerza la integración social e impide que no se agoten los recursos solidarios en las relaciones de los miembros de una comunidad.

b) Condiciones de participación: en otras palabras, la sociedad civil ofrece un marco para la acción de los individuos y comunidades pero esa acción debe aceptar la forma específica particular de ese contexto para poder participar. Esas condiciones consisten en la conciencia y el reconocimiento explícito de la relatividad de las propias representaciones de la "buena vida". Precisamente allí reside su pretensión cognitiva. Gracias a esto, se puede desarrollar una práctica política democrática aún bajo el peso de las diferencias étnicas, religiosas, culturales, etc. Esa política determina que los participantes deban reconocer formas alternativas de existencia como condición a su propia práctica política orientada a la realización de su ideal de la "buena vida".

En este análisis, la sociedad civil no sólo es el lugar donde los ciudadanos pueden y deben perseguir sus propios intereses particulares sino, por sobre todas las cosas, donde debe ser practicada intensamente la tolerancia en relación con las discusiones públicas.

A diferencia de la solidaridad, que se realiza y se practica sólo en la vida particular de las comunidades, la tolerancia se extiende en otra dirección. La misma demanda que las jerarquías y diferentes status al interior de la sociedad sean eliminados. Esto apunta a eliminar las fuentes o justificaciones para cualquier forma de discriminación. La tolerancia del pluralismo básico de las relaciones sociales es aceptar que existen otras versiones y formas de entender la propia cultura y religión pero no 
quita la posibilidad de defender la comprensión personal o de la comunidad de pertenencia de esa cultura o religión.

En esta línea de análisis, Walzer se distingue del resto de los comunitaristas que creen que la disminución de la integración y el progresivo carácter desvinculante de las relaciones sociales conduce a la absoluta desintegración de los lazos sociales. Para este autor, esta es la condición para que se desarrolle la tolerancia no sólo en la esfera política sino también en el seno de las comunidades plurales. En realidad, la tolerancia es una característica de las relaciones sociales de la comunidad que debe trasladarse a la esfera política.

La tolerancia tiene en la comprensión de Walzer un lugar mucho más amplio y políticamente más importante que en la tradición liberal. En dicha tradición, la tolerancia es la condición de la coexistencia pacífica de los distintos individuos bajo la protección del Estado. Es decir, la aceptación de opiniones y preferencias diferentes y discordantes con la propia. En cambio, para Walzer (1997, p.xi), la tolerancia representa el reconocimiento de las diferencias entre grupos y comunidades cuando sus lenguas, culturas, religiones o bien sus comprensiones del mundo y de la vida social difieren o resultan extrañas. Esta es la esencia y el contenido de la ciudadanía democrática. ${ }^{5}$ La tolerancia sirve para describir el comportamiento de reconocimiento recíproco de las diferencias de toda clase que pueden darse entre las comunidades (Höffe, 1988b, p.105-24).

Una sociedad civil tolerante demanda a las instituciones políticas que permitan y posibiliten que los distintos grupos puedan coexistir pacíficamente entre sí. Por supuesto, esta coexistencia pacífica puede adoptar diferentes formas políticas y cada una de ellas tienen implicaciones distintas para la vida moral de la sociedad. La mejor forma política o la más adecuada, depende en cada caso y para cada sociedad, de la historia, las tradiciones, y cultura de esa sociedad. ${ }^{6}$

Una forma de promover el desarrollo de una cultura de la tolerancia es diseñar un nuevo proceso de socialización de los individuos que fomente el ideal de coexistencia pacífica y de solución democrática de conflictos. Esta cultura es indispensable para no permitir que el lazo débil de la integración política de los ciudadanos no se rompa a pesar de

\footnotetext{
5 Krause \& Malowitz $(1998$, p.141) argumentan que el trato tolerante recíproco no implica ninguna clase de empatía.

6 Walzer piensa en este punto en el caso paradigmático de la sociedad americana, en donde la ciudadanía no cuenta con una historia y cultura comunes; y en donde, la práctica democrática se presenta como un lugar ideal para superar las diferencias entre los distintos grupos.
} 
los conflictos y que las costumbres civiles no se vean alteradas. Esa cultura debe asentarse en la tolerancia política pero sobre todo, debe fundarse en la tolerancia social de las comunidades. Fomentar el desarrollo de una conciencia positiva de la relatividad de la propia forma de vida es una necesidad de la práctica política de las sociedades avanzadas, sobre todo, de aquellas con población heterogénea y multicultural.

Al igual que otros comunitaristas, Walzer $(1997, \mathrm{p} .75)$ cree que esa re-socialización debe pasar por un cambio de orientación de la educación en las escuelas. Enseñar el pluralismo y desarrollar una conciencia positiva de la diferencia son esenciales para el desarrollo de la comunidad democrática. Por esto, rechaza que la educación del Estado social pretenda dar a los niños pertenecientes a comunidades menospreciadas o discriminadas, una conciencia positiva determinada de su pertenencia. La re-socialización no consiste en enseñarle a los individuos qué significa ser diferentes, sino transmitirle a aquellos cuya diferencia es discriminada, la manera correcta de ser diferentes. La idea central es generar un modelo de identidad comunitaria con autonomía propia.

Para esto, no sólo es necesario reformular la orientación de la educación oficial abandonando la cultura liberal del individualismo, sino que el Estado debe permitir que las comunidades sostengan sus propias escuelas, no como un reemplazo o una alternativa a la educación estatal sino como un complemento de la misma, tendiente a mejorar y fortalecer la autonomía de las comunidades y su fuerza integradora. Y esto debe entenderse como una incorporación a las prácticas democráticas, donde democracia es comprendida no como una mera forma de gobierno entre otras, sino una forma completa de vida, una experiencia común compartida con los demás.

El aporte de la educación al desarrollo y reproducción de esa forma de vida común radica en la capacitación de los individuos para participar de manera autónoma en la vida comunitaria. Y la vida democrática de las comunidades conforma la estructura social, política y cultural de la sociedad democrática. Por este motivo, el énfasis debe estar puesto en la formación de ciertas disposiciones para la acción social y no en la transmisión de una serie de valores o de un modelo de identidad determinado. En otras palabras, desarrollar la capacidad de interactuar moral y socialmente de la ciudadanía.

La sociedad civil, en tanto "esfera de esferas", es el lugar adecuado para realizar el ideal de una educación creativa que permita a los ciudadanos no sólo el desarrollo de disposiciones y capacidades individuales, autonomía y responsabilidad por los otros, sino también, “die Entwick- 
lung phantasievoller unbürokratischer und local wirksamer Lösungen zur Bewältigung gesellschaftlicher Probleme..." (Krauze \& Malowitz, 1998, p.145). ${ }^{7}$ Esas capacidades deben estar orientadas al mantenimiento y defensa de las formas democráticas de resolver los conflictos y a su vez, a la cooperación y al compromiso de los miembros de esas comunidades en las tareas relevantes de las mismas.

Esa capacidad debe ser entendida como una forma de virtud civil que sirve a la reproducción de la democracia como una auténtica forma de vida y que implica, como ya dijimos, una cierta competencia para interactuar moral y socialmente con el resto de la sociedad. Bajo las condiciones impuestas por el pluralismo étnico, cultural y religioso, la educación debe estar centrada en la tolerancia no en tanto instancia abstracta de las relaciones sociales sino como práctica concreta de las comunidades reales de la sociedad civil.

Michael Sandel (1992) considera que la moderna concentración y centralización del poder estatal se desarrolló en respuesta al crecimiento del capitalismo corporativo y de los mercados nacionales a fines del siglo XIX. Es decir, que el servicio de bienestar nacional se expandió para reemplazar los social bonds que el capitalismo estaba destruyendo. Pero las innovaciones en la creación de bienestar y la toma de riesgos en empresas individuales, fue reemplazada por un individualismo dependiente basado en demandas legales para la protección y el reconocimiento de derechos. La consecuencia de esto no fue solamente un debilitamiento del motor de crecimiento económico sino también un acelerado declinar de los sentimientos de obligación social y deberes sociales. Lazos comunitarios, kinship ties, etc. fueron reemplazados por garantías y seguros públicos otorgados y defendidos por grandes aparatos burocráticos diseñados para resistir las presiones democráticas from above (Anderson, 1988). Esas garantías que desplazaban los riesgos y se apoyaban en un avance del sistema legal sobre la comunidad, prometían reconciliar independencia respecto de los demás con seguridad frente a los riesgos del mercado y de la vida moderna. Esto permitía relevar a los individuos de la mutua dependencia y eliminar los dilemas morales de confianza, reciprocidad y obligación con los demás, sobre la base de un nuevo individualismo.

Más tarde, la respuesta del sistema a estos problemas, es decir, el reempowerment de los consumidores de servicios públicos intentó sensi-

7 Según estos autores, la concepción de resocialización de Walzer se basa en la obra de John Dewey y su idea de pedagogía democrática. 
bilizar a la administración burocrática de los bienes sociales frente a las necesidades personales de los recipients, pero la consecuencia fue una mayor despersonalización y heteronomía de la welfare provision. La presión por el mayor reconocimiento de derechos de bienestar ha continuado en aumento. Siempre según este autor, la iniciativa personal, la responsabilidad y la good-will en la provisión de bienes públicos, como salud, educación y servicios sociales profesionales prácticamente ha desaparecido. El aumento indiscriminado de derechos de bienestar creó una verdadera cultura de dependencia y minó los fundamentos morales del welfare state. La rethoric of rights ha desalentado la self reliance de los individuos y la ayuda mutua. Ha devaluado los lazos familiares y la red de asociaciones voluntarias que son un componente integrador esencial para la sociedad moderna. La cultura del individualismo dependiente ha destruido a las comunidades y sus funciones integradoras no se han visto reemplazadas ni recompuestas. La arista más saliente de este fenómeno de fragmentación es un aumento de la criminalidad, del desorden social, del vandalismo, etc.

Contra este diagnóstico, autores como Walzer y Etzioni proponen una resocialización de los individuos que pase por una nueva socialización de la responsabilidad individual. Etzioni cree que en este proceso resulta fundamental el rol que deben ocupar las familias para darle fundamento moral básico a la próxima generación. Los niños nacen con un moral vacuum que debe ser llenado por la familia con las normas morales necesarias para vivir en sociedad. En la actualidad, las sociedades sufren un parenting deficit gracias al cual, los niños crecen sin adecuados modelos morales a seguir, o directamente sin modelos claros. El resultado es una constante deficiencia de carácter, incapacidad para controlar impulsos y diferir las gratificaciones etc. (Etzioni, 1975, p.67-9). La integración social depende de una adecuada internalización de valores y normas sociales. En la actualidad, las familias dejan esa tarea en manos del sistema educativo de la educación pública que tienen la obligación de formar el carácter moral de los niños. Según este autor, el proceso de socialización debe asumir dos aspectos diferentes y complementarios:

1 La formación del carácter del individuo. Esto implica la adquisició$\mathrm{n}$ de rasgos personales básicos, como autodisciplina, auto-motivación, perseverancia, y capacidad para diferir gratificaciones. Estas características son fundamentales para tener una conducta moral correcta.

2 La internalización de commitents, es decir, de un conjunto de valores morales sustantivos que sólo se obtienen a través de una educación moral. 
Esta formación, que no puede quedar en manos del Estado, pretende sentar las bases para una integración social adecuada. Dicha integración debe desarrollar tres factores: a) rechazo de la violencia, b) lealtad a la democracia y c) respeto mutuo. Estos factores encarnan los valores centrales mínimos de una sociedad, necesarios para permitir la convivencia. $^{8}$

La simplicidad de este análisis, que lo expone a serias críticas, no debe engañar respecto a su profunda influencia en la política británica. Por lo pronto, supo inspirar el discurso de políticos, comentadores y funcionarios de gobierno que reconocieron rápidamente que una campaña basada en el declinar moral de la nación permitía explotar los miedos de los ciudadanos y generar un amplio caudal electoral. El propio primer ministro es un buen ejemplo de esto:

A solution to this desintegration doesn't simply lie in legislation. It must come from the rediscovery of a sense of direction as a country, and most of all from being unafraid to start talking again about the values and principles we believe in and they mean for us, not just as individuals but as a community. We cannot exist in a moral vacuum. (Mandelson \& Liddle, 1996, p.47)

Para contrarrestar las manifestaciones de desintegración social, Etzioni propone una moralización ciudadana basada en la recuperación del rol educativo de la familia a nivel de la comunidad, y en la retirada paulatina del Estado de esas funciones tradicionales.

Walzer también insiste en la necesidad de limitar ciertas funciones del Estado social en beneficio de la sociedad civil. Este autor no cree que la estructura anárquica de dicha esfera, sus diferentes agrupaciones y su sistema desigual de distribución de recursos pueda derivar en el predominio de un grupo determinado. La única instancia que por ser la suma de la fuerza pública presenta el peligro de desarrollar un exceso de poder es el Estado. En "Rescuing civil society", este autor recuerda que la sociedad civil puede ser un realm of inequality pero también lo es de self-help y de mutual aid, puede ser un realm of fragmentation pero también lo es de identity, puede ser un realm of fitfulness pero también lo es de engagement and activism. En este sentido, el Estado debe apoyar esa dimensión de la sociedad civil porque justamente allí se puede rescatar al Estado. Y

8 Etzioni especifica una serie de valores secundarios también importantes. Y si bien en ningún momento, habla de una acción concreta para la transmisión moral de esos valores, afirma que los mismos deben estar presentes en el conjunto de la educación de los individuos. 
la actividad de dicha esfera tiene también efectos directos en la inclusión social de los individuos:

that only a revival of associational life will produce a political revival, and only a political revival will produce the commitment (and eventually the ressources) necessary to overcome the inequalities of associationalism. Only the two together can bring excluded men and women into the common life. (Walzer, 1999, p.64)

El Estado en tanto instrumentalización del poder político no es una mera agrupación como cualquier otra. Es el contexto para la sociedad civil y no debe ocupar su lugar, sino generar un espacio para el desarrollo de las comunidades que la integran. Sociedad civil y Estado son dos elementos complementarios y necesarios para el desarrollo de una democracia moderna. Una sociedad civil activa y dinámica es el mejor contrapeso para un Estado poderoso. Y a su vez, un Estado democrático es el mejor espacio para el desarrollo de una sociedad civil democrática.

La sociedad civil es el lugar propicio para que los diferentes actores sociales persigan sus intereses. Las estructuras y las prácticas de la sociedad civil no pueden desarrollarse sin la mediación del Estado en los conflictos de las diferentes comunidades y en algunos casos, la protección de derechos de los individuos frente a las propias comunidades. La sociedad civil demanda del Estado no sólo su protección legal sino también un sinnúmero de otras actividades, sobre todo, aquellas dirigidas al bienestar social: subsidios para los programas de educación, rebaja de impuestos para las organizaciones privadas, reconocimiento jurídico para los intereses comunitarios, créditos estatales para las cooperativas, etc. Sin esa ayuda del Estado, la sociedad civil y las comunidades que la misma abarca y comprende, apenas podría subsistir. En esas relaciones con la sociedad civil, advierte Walzer, se encierra otro peligro potencial para las prácticas democráticas. Por este motivo, la ayuda estatal debe estar dirigida por la promoción de la justicia, brindando asistencia a las comunidades que se encuentran discriminadas u oprimidas por otras. Dicha ayuda debe darse a las comunidades que son discriminadas con el fin de que las mismas puedan ayudarse a sí mismas y no depender de la asistencia estatal. Por lo demás, el Estado debe limitarse a brindar el contexto para el desarrollo independiente de la sociedad civil y observar la más estricta neutralidad en el desarrollo de las diferentes formas de vida y actividades de la misma.

El Estado debe convertirse en un instrumento de apoyo y protección de aquellas agrupaciones y comunidades que son significativas para el 
mantenimiento del trato tolerante y las formas democráticas. Por este motivo, es fundamental que las instituciones del Estado social no se excedan en sus funciones buscando legitimación. ${ }^{9}$ Dichas instituciones nunca deben brindar asistencia a comunidades o agrupaciones que no lo requieran o deseen, o que no sean capaces de ayudarse a sí mismas (Walzer, 1992b, p.140-70). Esta debe ser la regla política con la que se debe decidir en cada caso, si la intervención del Estado social es necesaria o no.

El concepto de solidaridad es una de las categorías más antiguas del pensamiento político y moral. Su origen probablemente se remonta al derecho romano y su obligatio in solidum expresaba una forma de responsabilidad, en la que cada miembro de una comunidad era responsable solidario por las deudas de los demás. Recién a fines del siglo XVIII, esta categoría deja de vincularse a la noción de responsabilidad de deudas en el plano jurídico, para convertirse en una noción política, moral y social, que expresa ahora la obligación moral de reciprocidad entre los individuos y la comunidad. En el siglo XIX y de la mano de la revolución francesa, esta categoría es entendida en términos políticos como Fraternité. Más tarde, adquiere también la forma de un término técnico gracias al desarrollo de la sociología, y en la obra de A. Comte y E. Durkheim es caracterizada como un cemento social que sirve para la integración y la unidad social. En el siglo XX, autores como H. Bergson y M. Scheller la emplean en la filosofía moral en relación directa con categorías como Sympathie, Wohlwollen, Menschenliebe, Loyalität, o Gemeinsinn. Más allá de la evolución histórica del concepto y sus determinaciones heterogéneas, la idea central es que la solidaridad describe una unión recíproca entre los miembros de un grupo de seres humanos.

Ahora bien, en esa evolución, el concepto de solidaridad ganó una contenido normativo que opera por detrás de su uso habitual en la filosofía moral, en la política, en la sociología y en la vida cotidiana. Este contenido normativo tiene tres características relevantes:

1 Comunidad-La unión recíproca entre los miembros de un grupo no es solamente un hecho objetivo sino que debe ser asumido de ese modo por los miembros de ese grupo. Esto debe ser considerado seriamente y tener un significado subjetivo para los individuos. Los miembros deben identificarse con el grupo y sentirse emocionalmente unidos entre sí.

\footnotetext{
9 En este ítem se hace apenas un resumen de las críticas del comunitarismo al Estado social. Se exponen brevemente solo aquellas que tienen que ver con el problema de la integración social y la sociedad civil.
} 
2 Ayuda-Esa identificación y unión emocional dependen en gran medida de la espera de ayuda mutua en casos de necesidad y de la disposición de los demás para esa ayuda. Esta no tiene porque tener un fundamento altruista sino que puede ser entendida como una contribución al objetivo general de la comunidad y estar relacionada con la espera recíproca de ayuda en el futuro.

3 Legitimidad - La ayuda mutua y la contribución a la realización de intereses generales, generan legitimidad y mayor identificación con la comunidad.

En este sentido, la solidaridad debe ser analizada en relación con el concepto de nación, de sociedad y de Estado. Dentro de este contexto, la solidaridad es habitualmente vinculada a los lazos sociales derivados de un origen, una historia, una cultura, una forma de vida, ideales y destinos comunes. No casualmente en 1882, Ernest Renan definía a la nación como una comunidad solidaria. Esta idea tiene que ver con una renovación constante del lazo social que pasa por la renovación permanente del plebiscito que constituye políticamente a esa comunidad (Renan, 1995). Más tarde, y siempre dentro de la tradición francesa, H. Bergson acuerda que la nación se funda en distintos tipos de solidaridad cuya fuente es la naturaleza y los instintos humanos (Bergson, 1933). En realidad, estas caracterizaciones tampoco son nuevas. Ambas se apoyan en una interpretación particular de la tradición aristotélica, que coloca en la amistad el deseo de vida en común, es decir, que los hombres tendrían una naturaleza social que se expresa en esa "amistad", en tanto, forma de la solidaridad social. Entonces, el origen de las sociedades, se fundaría en la naturaleza humana que tiende a la sociabilidad en forma natural. Frente a esta tradición, autores como Kropotkin distinguían entre amor, simpatía e impulso social. Las dos primeras son una forma de las relaciones personales dentro de la sociedad, mientras que el impulso social, es la auténtica solidaridad, no personal sino humana, que sirve para fundamentar una sociedad de masas con una infinidad de relaciones anónimas (Kropotkin, 1975).

En la tradición alemana, F. Tönnies realizó una influyente distinción entre Gemeinschaft y Gesellschaft. La primera forma de agrupación humana es un grupo pequeño, familiar, unido por relaciones internas, lazos reales, es decir, wesentlich verbunden (esencialmente unidos). Mientras que la Gesellschaft, esta constituida por grupos grandes, anónimos, cuya unión tiene un carácter contractual, es decir, se trata de grupos wesentlich getrennt (esencialmente separados) (Tönnies, 1991). 
La pregunta que estos autores tratan de contestar es la siguiente: ¿Cómo es posible la unión de la sociedad? ¿Una sociedad, en términos de Tönnies, naturalmente separada?

El liberalismo ha contestado tradicionalmente esa pregunta a través del principio de la satisfacción de las necesidades humanas. Según este argumento, los individuos se unen y viven en sociedades para poder satisfacer mejor sus mutuas necesidades. Desde Hobbes hasta el neoliberalismo actual, la sociedad es un aglomerado de individuos, donde las relaciones sociales cumplen una tarea secundaria y son establecidas sólo para mejorar las posibilidades de realizar los propios intereses. La sociedad es un marco donde los individuos pueden perseguir sus fines personales y donde el mercado opera como el regulador más importante de la acción social y el Estado aparece como una instancia de protección.

En este punto, cobra importancia la obra canónica de E. Durkheim (1988) que consideraba que la solidaridad social era la auténtica y genuina fuerza integradora, que podía mantener unida a la sociedad. Esta solidaridad social se alimenta de dos fuentes distintas. Por un lado, de la solidaridad mecánica, que es una forma colectiva de solidaridad organizada a través de relaciones jurídicas. Por el otro, de la solidaridad orgánica que es resultado de la división del trabajo en la sociedad. Ambas modalidades existen una junto a la otra, y ambas conforman en distinta medida, la solidaridad social. Durkheim registraba en su época que existía una disminución constante de la solidaridad orgánica frente a un aumento permanente de la solidaridad mecánica. En este análisis, la evolución social cobra la forma de un proceso de caída de solidaridad, donde prima el egoísmo individualista y donde la relación entre los individuos deja de ser normativa y adquiere caracteres funcionales.

Esta pérdida de solidaridad es, en términos de Tönnies, el predominio del proceso de integración de la sociedad sobre la forma integradora de la comunidad. Ambos diagnósticos coinciden en señalar esa fuerza centrífuga del proceso de modernización que empuja a los individuos al aislamiento y que constituye una verdadera fuerza de separación, exclusión y desintegración. El individualismo creciente y el egoísmo individualista prevalecen frente a los intereses comunes, las creencias similares, los valores y la historia compartida, que cada vez más, juegan un rol marginal en los procesos de socialización de los individuos. Esto significa que las relaciones entre las personas dejan de ser personales y directas para convertirse en impersonales, indirectas y anónimas. Dichas relaciones ya no se fundan en afecciones personales sino en intereses complementarios e individuales. Cuando el proceso de socialización no transforma a los 
individuos en amigos sino en socios o en competidores, se fortalece la caída de la solidaridad. Esta es la dirección del Zeitdiagnose de Tönnies que ve la integración de la comunidades como un proceso natural y la integración de las sociedades como algo artificial y extraño a los individuos. La propia sociedad es un artefacto, un agregado mecánico de individuos frente al organismo vivo de la comunidad.

Este diagnóstico, que es una preocupación relativamente común dentro de la teoría social coincide con el comunitarismo, que retoma los motivos tradicionales de la crítica a la modernidad. Esta corriente de pensamiento apunta a recuperar las formas de la solidaridad social típicas de la comunidad, o al menos, las ideales. Frente al atomismo liberal de la sociedad moderna, Charles Taylor presenta la idea de una "solidaridad republicana" o bien de un "patriotismo" que a través de la defensa del bien común pueda superar los efectos del egoísmo de los individuos. La república, en términos de Taylor, se diferencia de la sociedad liberal por el tipo de unión que se da entre los miembros. Los habitantes de esa "república" están unidos por valores comunes y comprenden a la comunidad a través de un destino común integrador y por este motivo, están dispuestos a defender esa esencia comunitaria como un bien común directo. El patriotismo refiere a una determinada forma de identificación con los demás, basada en la participación de objetivos y empresas comunes. La república funciona como una forma de familia, en la cual, los integrantes sienten o pueden sentir el lazo de la solidaridad con los otros integrantes en sus objetivos comunes, en la forma común de expresar esos objetivos, en la forma común de exteriorizarlos, etc. La república es una familia en la medida en que una parte de ella, precisamente la que une a los individuos, está conformada por la historia común de esa república que los miembros sienten como propia y con la cual se identifican. La intensidad de los lazos familiares o de las viejas amistades se realimenta de manera permanente por la vida cotidiana común y refuerza los lazos que mantienen unida a la república a lo largo del tiempo y de los cambios de generaciones. En una república como la descripta, las diferentes familias y grupos sociales pueden tener diferentes destinos pero cada destino es un valor en sí mismo y forma parte del destino general de la república. Compartir ese destino común refuerza los lazos grupales y a su vez, fortalece el lazo de solidaridad de la república. En ese contexto, la obligación moral con el bienestar de los otros miembros es altruista pero forma parte del significado mismo del lazo social. El valor intrínseco de ese destino social es lo que le da sentido a esa unión y lo que llena también de sentido la relación particular con la comunidad y con las tareas comunes de la misma. Ese valor le da vida al patriotismo, o en palabras de 
Taylor a la virtu particular de los miembros de la misma (Taylor, 1993, p.103-30).

Por supuesto, la idea de una república como la imaginada por Taylor resulta muy interesante y quizás, aún puede tener la finalidad de ser un principio heurístico en la crítica social. Pero resulta difícil darle algún viso de realidad y en el fondo, presenta varios inconvenientes.

Para comenzar, es posible analizar el problema de las metas comunes. Walzer se queja continuamente de la falta de compromiso moral de los ciudadanos con el Estado. Estos ciudadanos necesitan del Estado pero no sienten ninguna lealtad, solidaridad, preferencia o afección por él. ${ }^{10}$ Taylor (1983) coincide al sostener que "the identification of a citizen with the republic, as a shared common enterprise, is essentially the recognition of a common good". Es esta identificación del ciudadano con la "república" la que genera un tipo de solidaridad que resulta fundamental para el buen funcionamiento de la sociedad. Una sociedad despótica necesita recurrir a la coerción o a la amenaza para obtener sacrificios o disciplina de sus ciudadanos. Según Taylor en una sociedad libre, el ciudadano acepta voluntariamente esos sacrificios y esa disciplina como una parte necesaria de la construcción del bienestar común.

Sin embargo, esta posición tiene varias desventajas. Por un lado, la imposibilidad de definir el bien común que puede generar esa identificación. La característica dominante de las sociedades actuales es el predominio de las relaciones instrumentales y anónimas en las cuales el llamado comunitarista a la familia resulta por lo menos ingenuo. La idea de los destinos comunes y compartidos también resulta irreal. En realidad, lo que predomina es la falta de acuerdo en las metas generales o directamente la falta absoluta de ellas, aún en las concepciones de los bienes públicos más imprescindibles y necesarios. Además, las dificultades para definir un bien común general, tiene también que ver con la enorme cantidad de posibilidades de vida que el proceso de modernización trajo aparejado. Este proceso no sólo aumentó la libertad de las personas sino que también aumentó sus posibilidades de llevar adelante formas de vida diferentes. La dificultad en definir un bien común general tiene un cos-

10 Quizás es posible establecer una diferencia entre autores como R. Dworkin o J. Rawls y M. Walzer. Para los primeros, el Estado debe ser neutral frente a los distintas concepciones de una "good life" mientras que el Estado en Walzer debe implicar el reconocimiento social de una concepción de terminada de "good life". Por esto es tan importante, el compromiso moral del ciudadano con el Estado. Para Walzer, una sociedad incluye una idea sancionada socialmente de lo que es o debería ser una "good life". Sin esto, no es posible hablar de comunidad o de cultura política. Vide Dworkin, 1985. También Rawls, 1983. 
tado positivo que es descuidado dentro del comunitarismo y tiene relación con ese aumento de la libertad individual para pensar y alcanzar formas de vida distintas. En las comunidades, añoradas por el comunitarismo, el bien común aparecía definido por tradiciones, historias, etc. pero en la actualidad, el debilitamiento de las mismas, le ha permitido a los individuos elegir con mayor libertad su propia concepción del bien común. Esto no significa solamente mayor libertad sino también mayor pluralismo.

Por otra parte, la formulación de Taylor pretende evitar el problema principal que arrastra la definición de ese bien común. Un Estado puede definir ese bien común en términos que coincidan con los de la ciudadanía y provocar por lo tanto adhesión, obediencia y aceptación general. Pero esto no dice nada de la moralidad de ese bien común o de la moralidad de esa definición. No se terminan de entender los motivos por los cuales una sociedad no podría generar identificación y participación de sus ciudadanos, sin necesidad de recurrir a la violencia y a la opresión aunque los fines de ese llamado a la identificación y participación sean inmorales. ${ }^{11}$

Por último, el comunitarismo no termina de explicar porqué la definición de esos bienes comunes o la existencia de los mismos, implica un aumento real de la solidaridad y de la integración social. Aquí aparece un implícito problemático en la teoría comunitarista, que sostiene que la existencia de bienes comunes a la sociedad aumentaría su integración y cohesión interna. Frente a esto, es posible argumentar que los bienes comunes tienen la facultad de unir a los miembros de una sociedad como también de separarlos. No existe ningún bien común, cultural social, religioso, etc. que no presente esta dualidad, o al menos, la posibilidad de tenerla. Ni siquiera una historia común, nos permite decir, que es un factor decisivo de integración social. La historia común une del mismo modo que separa y ninguna de estas posibilidades tiene a priori ninguna ventaja sobre la otra. Una historia común no sirve especialmente para que sus participantes definan intereses o bienes comunes o al menos tengan un destino común. Si así fuera, la historia de los conflictos políticos, sociales, etc. habría tenido un curso diferente. Ni siquiera los participantes de dichos conflictos o las víctimas de esas historias comunes tienen por lo general, destinos comunes.

11 La experiencia de los regímenes nacional-socialista en Alemania y fascista en Italia parecen apoyar esta idea. No resulta claro porque esa identificación tiene que ser necesariamente positiva. 
Por otra parte, esta recuperación de la comunidad y de los lazos solidarios implícitos en las mismas, deja de lado una apreciación histórica de la evolución social que no tiene porqué ser considerada en términos negativos. El proceso de modernización es leído unánimemente por el comunitarismo como una pérdida de solidaridad y una caída del lazo social. Pero en ningún momento, se interroga por el carácter de ese lazo social y de esa solidaridad. La evocación nostálgica de las comunidades antiguas deja de lado el carácter compulsivo que tenía la pertenencia de los miembros a esas comunidades. El propio Tönnies (1991, p.193) constata de manera indirecta este hecho relevando que la pertenencia a las comunidades premodernas tenía la forma de un destino histórico inamovible que de ningún modo, dependía de la voluntad de los miembros. El derecho primario de las comunidades era asegurar la permanencia de sus integrantes. ¿Qué tipo de solidaridad y qué carácter tenía esa solidaridad cuando la pertenencia a esas Zwangsgemeinschaften no era elegida, espontánea, libre, etc.? Es una pregunta que el comunitarismo no desea responder. De todos modos, la desaparición de esas comunidades y su particular sistema de solidaridades en manos del proceso de modernización, no debería ser considerado exclusivamente como una pérdida, sino al menos también, como una ganancia para el sistema de libertades de los individuos.

Otro problema que presenta el comunitarismo es una gran dificultad para definir el nivel político en el cual, los intereses de la comunidad estarían mejor representados. En este sentido, M. Sandel (1984, p.17), por ejemplo, cree que la acumulación de poder en la esfera federal de USA erosiona las bases naturales de las afiliaciones intermediarias, mientras que Walzer (1989, p.38-9) destaca la importancia de la soberanía nacional y del control de la inmigración como medios legítimos para preservar la cohesión y la integridad de los distintos grupos culturales. En Gran Bretaña, John Gray (1996) rechaza la idea de una política internacional y un proyecto económico integrado a Europa, insistiendo en la autenticidad de una cultura económica nacional y en la necesidad de protegerla de la homogeneización europea y de la irrupción del libre mercado.

Esta visión es un poco romántica y está alimentada por la creencia nostálgica en el Estado-nación, en tanto comunidad política basada en una homogeneidad lingüística y cultural, en la libre asociación y en el consenso. La defensa walzeriana de un control de la inmigración tiene que ver con la idea de que el Estado-nación es la precondición para la diversidad cultural interna, la distinción y la estabilidad de las tradiciones. Sin embargo, la historia del Estado-nación en occidente parece des- 
mentir esta idea. Precisamente, se trata de la historia de la homogeneización forzada de la cultura, religión y etnia, la sistemática negación de las historias particulares de la minorías y de las tradiciones secundarias. Lejos de producir ningún tipo de degeneración cultural o pérdida de tradiciones, el intercambio intercultural es un importante factor para el desarrollo cultural y para el enriquecimiento de las tradiciones (Bader, 1995). No es el aislamiento sino el complejo y dinámico intercambio con otras culturas, lo que permite la renovación de las propias tradiciones.

Retomando el tema del proceso de modernización, entendido en términos de un pasaje de las comunidades tradicionales a las sociedades modernas, ya vimos que el mismo no puede ser reducido a un mero proceso de pérdida de solidaridad, aún cuando este pasaje termina resultando en la disminución de un tipo determinado de solidaridad frente a otro (en términos de Durkheim). Si bien las condiciones actuales de la vida social están determinadas por relaciones anónimas e instrumentales donde predomina la persecución del interés individual, también es correcto afirmar que muchas formas de comunidad tradicionales han sobrevivido al proceso de la modernidad y se han desarrollado con pocos cambios.

Por otra parte, también se puede constatar que frente a la desaparición de algunas formas típicas de solidaridad, también han aparecido otras nuevas en su reemplazo. Estas nuevas formas se diferencian de la tradicionales en varios sentidos. Por lo general, se trata de solidaridades puntuales, es decir, restringidas en el tiempo y limitadas a un aspecto de la persona. No son solidaridades que relacionan para toda la vida a una persona sino que dan la libertad de cambiarla o darle nueva forma al compromiso. Esta solidaridad libremente elegida y no compulsiva como la de las sociedades premodernas, le da al individuo la posibilidad de renovar el lazo si las relaciones sociales de la comunidad corresponden a sus deseos (Giddens, 1991). Estas formas débiles de solidaridad están marcadas por el respeto a la autonomía del individuo, que decide en forma autónoma entrar a la comunidad y en forma autónoma permanecer en ella. Un buen ejemplo son los grupos de auto-ayuda y las iniciativas ciudadanas, siempre restringidos en el tiempo y que rara vez conciernen a la persona en su totalidad sino sólo en un aspecto determinado de su vida social. El pasaje de la comunidad a la sociedad como marco para las relaciones sociales, también ha tenido un efecto de "cosmopolitización" de dichas relaciones. En efecto, mientras que en las comunidades premodernas, los individuos ajenos a la comunidad era vistos como extraños y como posibles enemigos, la socialización moderna, el aumento de la 
población y la consiguiente aparición de la sociedad de masas, ha provocado la desaparición de esas formas tradicionales de exclusión. En la actualidad, la vida social de las personas está poblada precisamente de "extraños" que es imposible evitar y excluir. Los "extraños" han pasado a convertirse solamente en "alguien que no conozco" (ibidem). Esta superación de los particularismos, de las relaciones regionales a favor de una dimensión más abarcadora, pluralista y universal, probablemente se deba al surgimiento del Estado-nación en el siglo XIX, e implica un grado de integración social abstracto pero no por eso, menos importante o con menos consecuencias para las relaciones sociales de los individuos (Habermas, 1999b, p.128-53).

En su análisis del proceso de modernización, el comunitarismo deja de lado explícitamente estos costados positivos. Frente a la caída de lazos fuertes de solidaridad, han aparecido otros lazos solidarios que respetan la libertad y autonomía de los individuos; frente a la pérdida de destinos comunes que sirven para orientar la acción social de la comunidad, han aparecido formas de libertad de acción más completas para las personas; frente a la caída de las comunidades tradicionales premodernas, se constata un aumento de la libertad de las personas que ya no están determinadas por su pertenencia a una comunidad, sino que pueden elegir libremente entre cualquiera de ellas, la que más cercana se encuentre a su forma de entender la "good life". Posiblemente, este descuido se deba a que el comunitarismo orienta su análisis social por la crítica ideológica de la cultura liberal. Entonces, la pérdida de objetivos comunes, la caída de las viejas formas de comunidad y el debilitamiento de los antiguos lazos solidarios sólo son vistos en el marco del aumento de la comprensión individualista de la sociedad y de sus consecuencias negativas para la integración social. La conclusión comunitarista es que el individualismo no proporciona ninguna base para los problemas de integración, estabilidad política y cohesión social. El Estado social y la sociedad son vistos por los individuos como meros instrumentos para alcanzar sus fines individuales. En relación con esto, Walzer (1989, p.63) reseña el débil lazo moral que une a los ciudadanos con el Estado:

Its protagonists conceive of citizenship in economic terms, so that citizens are transformed into autonomous consumers, looking for the party or program that most persuasively promises to strengthen their market positions. They need the state but have no moral relation to it, and they control its officials only as consumers control the producer of commodities, by buying or not buying what they make. 
El modelo de ciudadano descripto requiere del Estado para poder realizar sus fines personales pero no siente ninguna solidaridad, sentimiento o relación especial con ese Estado. Esta situación sumada a la desaparición de valores comunes, de la base moral de la esencia comunitaria y la caída de los sentimientos solidarios permite decir que el sistema democrático está en peligro. El comunitarismo se refiere aquí a la gobernabilidad política de las sociedades que presentan estos síntomas de desintegración social. Sin embargo, en términos políticos, la integración de un grupo social o la falta de ella, tiene gran importancia, más allá de las consecuencias de todo tipo para las personas, en la esfera de la instrumentación de políticas de gobierno. Es decir, en referencia a los problemas habituales de legitimidad del sistema político, obediencia del derecho, etc.

En este sentido, las soluciones propuestas por el comunitarismo para detener el proceso de desintegración social, no pasan por transformaciones en el sistema político, en el jurídico o económico, a fin de promover una democratización de dichos sistemas que tenga efectos integradores en el sistema social, es decir, cambios que generen legitimidad real en la ciudadanía. Excepto en el caso de Walzer, que promueve una democratización de la esfera política a fin de devolver el control real de los mecanismos políticos de gobierno a los ciudadanos, ${ }^{12}$ el resto del comunitarismo cree que la solución a la desintegración social hay que buscarla en el plano social.

La argumentación de Taylor por una solidaridad republicana o por un "patriotismo republicano" 13 hace referencia precisamente a esta preocupación por el sistema democrático, la libertad y la autonomía de las personas. En este análisis, la relevancia política de la solidaridad y el patriotismo son bastante claras. Para Taylor, el patriotismo es una condición importante para posibilitar la libertad. Una sociedad fundada solamente en el interés, en la mera la persecución de fines individuales no constituye ningún entramado político suficientemente fuerte para contrarrestar los peligros que debe asumir la democracia. Una sociedad como

12 El programa político de Walzer (1994) tiene los siguientes puntos: 1. Democratización radical de las corporaciones gubernamentales. Esto significa trasladar la dimensión de la toma de las decisiones económicas a la esfera pública y convertir esas decisiciones en parte habitual de la discusión de los asuntos públicos. 2. Descentralización de la actividad gubernamental. Esto significa alterar la escala habitual de la vida política y aumentar el número de ciudadanos capaces de tener un rol efectivo en la toma de decisiones diaria de la política. 3. Creación de partidos y movimientos sociales que puedan operar en diferentes niveles de gobierno y puedan generar un grado mayor de compromiso individual que los actuales partidos nacionales.

13 Taylor (1993, p.122) usa ambos conceptos como sinónimos. 
la descripta no podría jamás movilizar a los ciudadanos a enfrentar u oponerse a la amenaza real de potenciales déspotas o tiranos, por ejemplo. En un caso así, habría muy pocas personas que serían capaces de arriesgarse para defender los principios universales que rigen dicha sociedad. Individuos libres de toda identificación normativa con la polis kantiana ideal que propone el liberalismo, tendrían y sentirían muy pocas motivaciones para resistir un tirano, si éste les ofrece la protección de sus propiedades y riquezas y seguridad personal. ¿Sobre la base de qué identificación y apelando a qué valores puede ser movilizada la ciudadanía, cuando dicha institución no se basa justamente en ninguna identificación ni en ningún valor particular?

En resumen, para Taylor, solidaridad y patriotismo son partes constitutivas de una sociedad libre y democrática. Ahora bien, ¿cómo podemos hacer para detener su caída o desaparición? La respuesta del comunitarismo es, en todo caso, bastante clara y pasa casi exclusivamente por la dimensión social de este problema. Si la desaparición de las comunidades y la falta de solidaridad en las sociedades modernas es consecuencia del individualismo, resulta lógico que se considere que alcanza con una reforma ideológica de la conciencia para detener el proceso y revertirlo.

Ya vimos en la primera parte que Walzer y Etzioni hablaban de una resocialización moral de los individuos a fin de desarrollar la formación de ciertas disposiciones para la acción social, es decir, la capacidad de interactuar moralmente en la sociedad. Esta resocialización provocaría una nueva unificación de la sociedad.

En la misma dirección, apuntan los trabajos de Taylor y también de MacIntyre (1988, p.36) que menciona la necesidad de una conversión a través de la cual, sea posible superar la cultura del liberalismo. Todo el comunitarismo acuerda con esa necesidad. Sólo podemos encontrar diferencias en las modalidades de realización de ese cambio de conciencia, es decir, en el carácter que debe tener el proceso de resocialización de las personas. Dicho proceso puede ser entendido de formas diferentes, según los distintos autores, desde una reforma "desde abajo" hasta una reforma "desde arriba" desde los propios mecanismos del Estado. Etzioni presenta un programa político completo que va desde cambios educativos hasta cambios en el derecho familiar que no permitan a las parejas casadas separarse con facilidad, para presionarlas a la estabilidad y la permanencia, cambios que no permitan a las parejas separadas dejar en manos del Estado la responsabilidad por sus hijos, etc. Por ejemplo, Fletcher (1994, p.167) propone que "daß wir Klassenzimmer als Vehikel verwenden, um loyale und patriotische Empfindungen einzuflößen" y el ejercicio de cierta censura legítima. 
El programa comunitarista, que propone una reforma de conciencia para solucionar los problemas de desintegración social, parece asentarse en varios prejuicios. Para comenzar, resulta absolutamente naiv creer que los problemas de integración se originan en el plano intersubjetivo, en donde, las creencias personales, prejuicios, etc. operan y donde una "reforma de conciencia" tendría algún sentido. Este prejuicio iluminista consiste en pensar que los individuos reaccionarían siempre racionalmente en su vida social si conociesen los motivos de su propia acción y las causas de los problemas sociales, es decir, si tuviesen la educación adecuada. La idea general es que los problemas de exclusión y discriminación no tienen causas estructurales fundadas en la economía o en la dimensión política, sino que están causados por "falsas comprensiones" de las diferencias en las distintas formas de vida. Si a través de una nueva educación, podemos disolver esas "falsas comprensiones" vamos a poder solucionar los problemas sociales. Esta forma de entender la crítica social reduce los problemas de la sociedad, en este caso, la acción de factores de desintegración a problemas de conciencia. No hay una dimensión económica, ni política que empujan esos factores provocando la aparición de las "movilidades" walzerianas. ${ }^{14}$

Por otra parte, el comunitarismo parece basar su programa de integración social en las mismas premisas individualistas que la cultura liberal propone para el análisis social. Esa "reforma de conciencia" provocaría un cambio en las acciones de los individuos que en algún momento se transformarían en acciones sociales. La idea es que la acción social parece ser la suma de las acciones de los individuos que la componen. Precisamente esta es la idea central de la cultura liberal: una sociedad es solo la suma de sus individuos. La realidad social está determinada por la suma de las acciones individuales sin que intervengan factores causales estructurales. Por este motivo, el comunitarismo puede creer que un cambio de conciencia se traduciría de inmediato en un aumento de la solidaridad social y del pluralismo y por consiguiente en una reversión del fenómeno de desintegración social. Por eso, no cree necesario explicar el modo en que un cambio de conciencia debería reflejarse o se transformaría necesariamente en cambios sociales.

14 Desde el punto de vista de la integración social, Michael Walzer (1990) se ha encargado de reseñar en The Communitarian Critique of Liberalism, los cuatro factores más importantes causantes del proceso actual de desintegración de los lazos sociales y de ruptura de los vínculos de pertenencia. Este proceso de fragmentación social que aparece en las sociedades altamente desarrolladas se encarna en cuatro aspectos distintos del proceso de modernización y erosiona en múltiples formas los social bonds de los individuos con su lugar de pertenencia, con su grupo social, con su familia y con su comunidad política. 
Analicemos ahora brevemente la propuesta de integración social de Etzioni, que probablemente haya sido el único autor comunitarista cuyas ideas se hayan visto realizadas o al menos impulsadas directamente por un programa político de gobierno..$^{15}$ Este autor enfatiza las virtudes que una educación moral debería transmitir:

We need to return to a society in which certain action are viewed as beyond the pale, things that uprigth people would not do or even consider: to walk out on the their children, file insurance claims, cheat on tests. (Etzioni, 1975, p.24)

Por supuesto, otros vicios también son fuertemente condenados:

chronic malingering at works and workers calling in sick when they are not sick, driving whitout a licence, failing to observe the speed limit, refusing to pay taxes or serve on a jury, stealing, lying, and other expressions of today's deficient work ethics. (ibidem, p.27)

Entonces, las virtudes que debe transmitir la educación moral deben ser el auto-control, la veracidad y la convicción de que hard work pays. Sin embargo, estas virtudes no parecen ser fundamentales para la autonomía moral de los individuos sino para la integración funcional de la sociedad y en todo caso, para aumentar su eficiencia económica.

Workers need such self-control so that they can stick to their tasks rather than saunter into work late and turn out slapdash products, becoming able to observe a work routine that is often not very satisfying by itself. Citizens and community members need self-control so that they will not demand ever more services and handouts while being unwilling to pay taxes and make contributions to the commons. (p.91)

El proceso de resocialización de los individuos parece tener la función de aprender a diferir la gratificación y a desarrollar las cualidades

15 Ya vimos que dicha propuesta fue adoptada casi oficialmente por la New Labour en Gran Bretaña. Autores como Callinicos (1999), afirman que el discurso comunitarista coincide plenamente con el programa político del Third Way (Blair, Clinton, Jospin, Schröder). Dicho autor define este programa del siguiente modo: "The radical centre, the new democratic state, active civil society, the democratic family, the new mixed economy, equality as inclusion, positive welfare, the social investment state, the cosmopolitan nation, the cosmopolitan democracy". En este artículo, Callinicos considera que el acuerdo fundamental entre comunitarismo y Third Way es la redefinición de igualdad entendida como inclusión. Y la inclusión es lograda desarrollando políticas que persigan el aumento del sentimiento de pertenencia de los individuos. 
que son socialmente útiles y moralmente apropiadas. Sin embargo, el problema de este planteo es que confunde utilidad social y autonomía moral.

La moralidad consiste en tomar responsabilidades, por nuestras acciones, significados y consecuencias. El pasaje a la modernidad significó también el reemplazo de la autoridad absoluta y las relaciones personales de obediencia y dominación por jerarquías impersonales y formas legales formales de coordinación de la acción social. Estas formas permiten también el control de las diferentes esferas de la vida social. Este pasaje eliminó muchas de las tradicionales modalidades de poder y dependencia. Una consecuencia fue una ganancia moral directa para el individuo moderno. Los miembros de las sociedades postindustriales tienen un sentido moral más profundo y un sentido de la responsabilidad más extendido que los de las comunidades premodernas. Pero el problema es que los mecanismos formales de integración funcional que han provocado esta liberación de las personas de los antiguos sistemas de dominación, se han expandido y lo siguen haciendo, al punto de poner en peligro la autonomía de ciertas esferas de vida cuyo sentido requiere la auto-determinación (solidaridad social, por ejemplo) y no los mecanismos funcionales de integración.

Las obligaciones sociales formalizadas necesarias para el funcionamiento de sistemas sociales altamente diferenciados están diseñadas para obtener ciertos resultados que las personas no pueden alcanzar individualmente. Dichas obligaciones obtienen legitimidad a través de los resultados a los que apuntan. Esas metas son la protección y la realización de la libertad, responsabilidad y pleno desarrollo de los individuos. Esas obligaciones que demandan obediencia los sujetos además de su cumplimiento, requieren de los mismos el compromiso y el sacrificio de su autonomía y son el medio para alcanzar los fines de libertad, responsabilidad, etc. El peligro es que esos medios que pretenden proteger la libertad terminen limitándola innecesariamente. Esta paradoja inhabilita el sistema de valores que propone Etzioni para la resocialización.

Por otra parte, si es correcto pensar que la cultura liberal ha desconectado los resultados en términos sociales de pagar impuestos, trabajar duro en el empleo, obedecer la ley, etc. con nuestras metas personales, valores e intenciones, no se entiende cómo la educación moral puede volver a poner en contacto estas esferas. Si las relaciones entre los individuos está mediada exclusivamente por las fuerzas del mercado y las regulaciones administrativas y legales, las obligaciones sociales mencionadas anteriormente tienen legitimidad política pero no moral. Y por este motivo, ninguna educación puede refundarlas moralmente sin caer en contradicciones. 
Las obligaciones sociales funcionales obtienen legitimidad a través de la esfera política pero no adquieren nunca relevancia moral para los individuos. Si la sociedad democrática moderna pretende preservar la autonomía de sus miembros, no debe buscar la consecución de las obligaciones sociales a partir de la moralización de la conformidad de los ciudadanos. Por el contrario, los sistemas sociales obtienen legitimidad en una esfera política abierta a través de la convicción pública renovada constantemente. Estas obligaciones deben ser aceptadas porque están subordinadas a objetivos humanos, es decir, a un propósito social y no a sí mismas.

Esta falla del planteo comunitarista en distinguir entre las regulaciones funcionales de la conducta diseñadas para alcanzar fines sociales que no pueden ser alcanzados individualmente y la auto-determinación de las relaciones sociales reguladas por individuos responsables y reflexivos se extiende también a sus consideraciones respecto del trabajo. Etzioni y otros comunitaristas como Bellah (1985, p.287-8) proponen rehabilitar el sentido ético del trabajo. En términos generales, promueven la reapropiación del trabajo a través de un sentido religioso o moral, en el cual, los individuos pueden encontrar verdad, autenticidad y purificación moral a través de la dignidad que aporta el trabajo social. Etzioni (1975, p.113) cree que un año de servicio social para los jóvenes que dejan la escuela serviría para disminuir el desempleo y la criminalidad, y al mismo tiempo "build up the moral tenor and sense of social responsability amoung the young".

En este punto, se apuesta a revivir el mito del trabajo que paradójicamente las sociedades avanzadas altamente diferenciadas y sus mecanismos seculares de compensación, castigo y recompensa, han vaciado por completo de credibilidad. El trabajo ya no cuenta en nuestros días con ningún contenido moral. Más allá de esto, el comunitarismo vuelve a confundir las obligaciones funcionales con los commitments personales, deberes sociales formales con lazos sociales voluntarios. El consentimiento a las regulaciones sociales y al trabajo social no debe estar relacionado directamente a la responsabilidad moral, a la autonomía creativa o a la renovación espiritual de la sociedad. El respeto a las regulaciones funcionales del grupo social deben ser entendidas como una restricción de la libertad y la autonomía de los individuos a favor de objetivos sociales que son fair y benéficos para todos.

Estas consideraciones del comunitarismo parecen dejar de lado otro problema general referente a la solidaridad. Y es la posibilidad de contar con una sociedad absolutamente egoísta, es decir, compuesta por indivi- 
duos que sólo persigan sus intereses particulares, pero justa. Esto significa plantear la posibilidad teórica de una sociedad con bajos niveles de solidaridad pero en la cual se realicen los principios generales de justicia con los que el propio comunitarismo acuerda. En teoría, al menos, la justicia (Habermas, 1999a) o el cumplimiento efectivo de la misma y sus consecuencias políticas y sociales podría tener un efecto integrador mucho más fuerte que la solidaridad cuyo signo moral no aparece en principio determinado por la constitución de un bien común para el conjunto de la sociedad. En cambio, la justicia y sus efectos igualadores en el trato, en el reconocimiento de la dignidad de las personas, etc. puede ofrecer una perspectiva real de integración no sistémica ni meramente funcional. Más allá del egoísmo individual, y de la falta de solidaridad, la igualdad propuesta por la justicia presenta un lugar común donde los individuos pueden sentirse unidos a los demás. En este sentido, la justicia y su demanda de realización a través de los derechos ciudadanos en la institución de ciudadanía ${ }^{16}$ parece plantear, en muchas formas, una alternativa real a la promoción de la solidaridad del programa comunitarista como factor de integración social (Lukes, 1998).

FONDEVILA, G. The function of the tolerance in the resocialization of the citizenship. Trans/Form/Ação (São Paulo), v.24, p.183-212, 2001.

- ABSTRACT: This papers analyzes the communitarian project of social integration exposed in the Walzer and Etzioni's work as a possible answer to the disintegration of social bonds in the advanced societies (family breakdown, unchecked criminality and social disorder). It deals with the notions of tolerance, solidarity and moral resocialization of the citizenship. In this sense, some considerations are made about the functional character deduced of this communitarian model of social integration and show the extent and limits of this model (solidarity and tolerance).

- KEYWORDS: Communitarianism; resocialization; tolerance, solidarity; social integration.

16 ¿No era esta acaso, la idea original de los derechos sociales, según Marshall? Ver Marshall, 1992. 


\section{Referências bibliográficas}

ANDERSON, K. et al. Roundtable on communitarianism. Telos, n.76, Summer 1988.

BADER, V. Citizenship and exclusion. Political Theory, v.23, n.2, 1995.

BELL, D. The Cultural Contradictions of Capitalism. New York: Basic Books, 1976.

BELLAH, R. et al. Habits of the Heart. Berkeley: University of California, 1985.

BERGSON, H. Die beiden quellen der moral und der religion. Jena: 1933.

CALLINICOS, A. Social theory put to the test of politics: Pierre Bourdieu and Anthony Giddens. New Left Review, n.236, Jul.-Aug. 1999.

DURKHEIM, E. Über soziale Arbeitsteilung: Studie über die Organisation höherer Gesellschaften, Frankfurt: s.n., 1988. [Título original: De la division du travail social: étude sur l'organisation des societés supérieures. Paris, 1933.].

DWORKIN, R. A Matter of Principle. Cambridge: s.n., 1985.

ETZIONI, A. The Spirit of Community. London: s.n., 1975.

FLETCHER, G. P. Über die Moral von Beziehungen. Frankfurt, 1994.

FRANKENBERG, G. Civil society and social justice. In: WALZER, M. Toward a Global Civil Society. Providence: s.n., 1995. p.195-8.

GIDDENS, A. The Consequences of Modernity. Cambridge: s.n., 1991.

GRAY, J. After Social Democracy. London: s.n., 1996.

HABERMAS, J. Die Krise des Wohlfahrtsstaats und die Erschöpfung utopischer Energien. Frankfurt: s.n., 1985.

. Die Einbeziehung des Anderen. Frankfurt: Suhrkamp, 1999a.

. Der europäische Nationalstaat: Zur vergangenheit und Zukunft von Souneranität und Staatsbürgershaft. In: Andersen. Frankfurt: Suhrkamp, 1999b.

HÖFFE, O. Den Staat braucht selbst ein Volk von Teufeln. Philosophische Versuche zur Rechts: und Staatsethik. Stuttgart: s.n., 1988a.

. Pluralismus und Toleranz: Zur Legitimation der Moderne. In: Den Staat brancht selhst ein volk von tenfeln. Philosophische Versuche zur Rechts: und Staatsethik. Stttgart: s.n, 1988b.

HONNETH, A. Kampf um Anerkennung. Frankfurt: s.n., 1992.

KRAUSE, S., MALOWITZ, K. Michael Walzer zur Einführung. Hamburg: s.n., 1998.

KROPOTKIN, P. Gegenseitige Hilfe in der Tier: und Menschenwelt. Berlin: s.n., 1975. 
LUKES, S. Solidarität und Bürgerrechte. In: BAYERTZ, K. Solidarität: Begriff und Problem. Frankfurt: s.n., 1998. p.389-98.

MACINTYRE, A. Whose Justice: which rationality. Notre Dame: s.n., 1988.

MANDELSON, P., LIDDLE, R. The Blair Revolution. London: s.n., 1996.

MARSHALL, T. Citizenship and Social Class. London: Pluto Press, 1992.

RAWLS, J. Theory of Justice. Cambridge: s.n., 1971. . Justice as fairness: political not metaphysical. Philosophy and Public Affairs, v.14, n.3, p.223-51, 1983.

RENAN, E. Qu'est-ce que c'est une nation? Paris: s.n., 1995.

SANDEL, M. Morality and the Liberal Ideal. The New Republic, 1984. p.17. . The procedural republic and the unencumbered self. In: AVINERI, S., SHALIT, A. Communitarianism and Individualism. Oxford: s.n., 1992, p.45-56. Tagesspiegel, 23.5.1999, p.8-23.

TAYLOR, C. Cross-purposes: the liberal communitarian debate. In: ROSENBLUM, N. Liberalism and the moral life. Cambridge: s.n., 1983. . Sources of the Self: the Making of the Modern Identity. Cambridge: s.n., 1989.

The Etics of Authenticity. Cambridge, 1991.

. Aneinander vorbei: Die Debate zwischen Liberalismus und kommunitarismus. In: HONNETH, A. (Ed.) Kommunitarismus. Eine Debate über die moralischen Grundlagen moderner Gesellschaften. Frankfurt: s.n., 1993. p.103-30.

TÖNNIES, F. Gemeinschaft und Gesellschaft. Grundbegriffe der reinen Soziologie. 3 Univeranderte Aufl. Darmstadt: Wissenschaftliche Buchgesellschaft, 1991.

WALZER, M. Dissatisfaction en the welfare state. In: Radical Principles. Reflections of an Unreconstructed Democrat. New York: s.n., 1980. p.23-53.

. Spheres of justice. Oxford: s.n., 1989.

. The communitarian critique of liberalism. Political Theory, n.1, 1990.

. Zivile Gesellschaft und amerikanische Demokratie. Berlin: s.n., 1992a.

. Ethnischer Pluralismus und politische Demokratie. In:___. Zivile Gesellschaft und amerikamische Demokratie. Berlin: s.n., 1992b.

Civility and civic virtue in contemporary America. In: TURNER, B., HAMILTON, P. Citizenship. London: Routledge, 1994. p.176-87.

. On Toleration. New Haven: s.n., 1997.

Rescuing civil society. Dissent, Winter, 1999. 